\title{
Meta-analysis: Statistical Trickery or Sound Science?
}

\section{Zahra Sohani}

\begin{abstract}
The goal of a systematic review is to present a balanced summary of existing research. In order to accomplish this, systematic reviews include a thorough search of relevant articles, including published and unpublished, using explicitly defined and reproducible criteria. The main rationale for conducting a meta-analysis comes from the fact that combining individual studies provide an increased sample size, which consequently improves the statistical power to detect treatment effect. If all steps outlined are followed properly and authors remain transparent regarding the design and conduct of the metaanalysis, this technique provides an excellent and scientifically sound means of synthesizing evidence.
\end{abstract}

Keywords: Meta-analysis, Systematic review, Quality, EBM.

How to cite this article: Sohani Z. Meta-analysis: Statistical Trickery or Sound Science? J Postgrad Med Edu Res 2012; 46(1):12-14.

\section{Source of support: Nil}

Conflict of interest: None declared

\section{WHAT IS A META-ANALYSIS?}

In many medical disciplines, it is common to find several research studies that seek to answer similar questions or duplicate results found previously. However, these studies tend to vary in quality and may report conflicting results. In order to adequately answer the research question, it is important to regard the range of information available on that topic. Meta-analysis is 'a statistical procedure that translates results from different studies to a common metric and statistically explores differences in study characteristics and findings'. ${ }^{7}$ A well-conducted meta-analysis will present an objective appraisal of current evidence and provide a precise estimate of the treatment effect. ${ }^{4}$

Systematic reviews are at the heart of meta-analyses. The goal of a systematic review is to present a balanced summary of existing research. In order to accomplish this, systematic reviews include a thorough search of relevant articles, including published and unpublished, using explicitly denied and reproducible criteria. In a properly conducted systematic review, research designs and study characteristics are appraised and the results are synthesized. ${ }^{3}$ Using the studies included in the systematic review, an estimate of the summary effect is provided-this estimate is attained through a meta-analysis. ${ }^{9}$

\section{Effect Size}

The primary goal of meta-analysis is to calculate a summary effect size. Individual studies commonly present their results as a frequency of an outcome, such as stroke or death. ${ }^{9}$ For meta-analysis, these frequencies are converted to summary measures. If the outcome is binary (e.g. disease vs no disease) then odds ratios or relative risks are the measures used. If the outcome is continuous (e.g. blood sugar measurement) then mean differences are used ${ }^{4,5}$ Odds ratio (OR) is defined as 'the ratio of the odds of the intervention group to the odds of a control group'. Odds are a quotient of the number of patients in the group who achieve a certain end point and the number of patients who do not. Risk, in contrast to odds, is calculated as the number of patients in the group who achieve the stated end point divided by the total number of patients in the group. ${ }^{1}$ Relative risk (RR) is the ratio of the two risks. An RR or OR less than 1 indicates decreased likelihood of outcome in the intervention group, a ratio greater than 1 indicates increased likelihood, and a ratio of 1 indicates no difference. ${ }^{1}$

The overall treatment effect is calculated using different models based on the heterogeneity of the data. Heterogeneity and the corresponding models used are discussed later in this article. The results of a meta-analysis are commonly depicted using forest plots. An example is shown in Figure 1 below adapted from Akobeng (2005). ${ }^{1}$ The black squares on this plot represent the ORs of individual studies with their 95\% confidence intervals (CI). The area of the black square is representative of the weight of each study (usually proportional to sample size). The overall treatment effect is depicted by the diamond often found at the bottom of the plot, the center of which represents the combined effect with its $95 \%$ CI. ${ }^{1}$

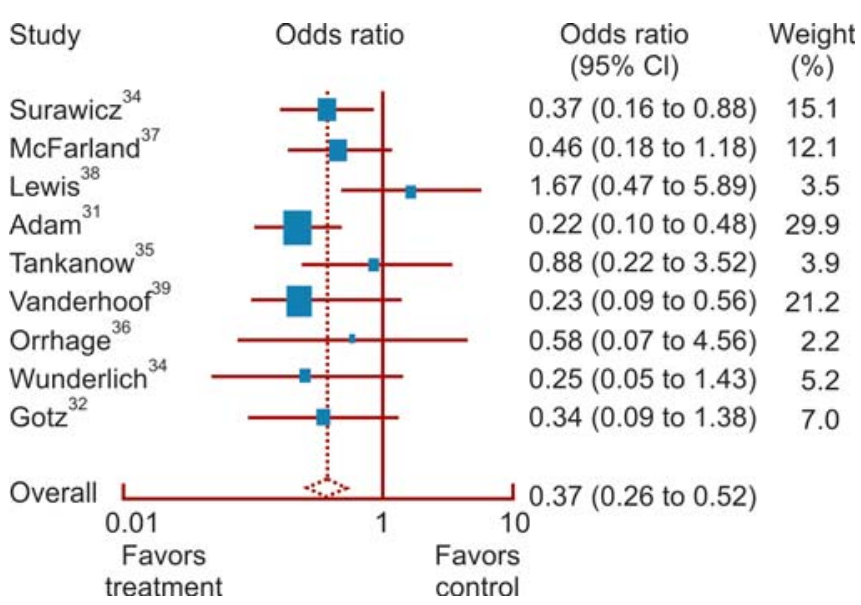

Fig. 1: Example forest plot to depict summary measures in meta-analysis 


\section{ELEMENTS OF META-ANALYSIS}

The main rationale for conducting a meta-analysis comes from the fact that combining individual studies provide an increased sample size, which consequently improves the statistical power to detect treatment effect. ${ }^{1}$ Additionally, meta-analysis can detect biases and inadequacies in design, conduct, analysis and/or interpretation of the included studies. The information gathered from meta-analysis can be used to encourage improvements in the quality of the research necessary to 'optimize' medical care. ${ }^{10}$

The greatest strength of meta-analysis is the transparency with which all the decisions relevant to achieving the final composite effect size are depicted. These processes are discussed below.

\section{Quality Analysis}

Combined results of a meta-analysis are no more reliable than the quality of the studies on which they are based. Wellconducted meta-analyses use explicit and objective criteria for inclusion and exclusion of the studies through which readers can assess the quality of the data included. ${ }^{9}$ Quality involves various factors, including design, conduct and analysis of the study. ${ }^{6}$ Numerous checklists are available to assess the quality of the studies included. Two commonly used are those developed by Jadad et al and Chalmers et al. ${ }^{9}$ By incorporating study quality into a meta-analysis, trials that fail to meet a certain standard of quality will be excluded, thereby ensuring the use of quality data.

\section{Heterogeneity}

If results of individual studies differ greatly, combining them may not be appropriate. Naturally, to get a precise answer to a specific question, only studies that attempt to answer that exact question should be included. Studies can differ with respect to the population sample, the methods used to assess the end point, the treatment, and the primary end point itself. ${ }^{9}$ These systematic differences between studies can influence the effect size. The central difficulty lies in deciding which studies are 'combinable'. In order to ascertain this, meta-analysis can test for the existence of heterogeneity. While many such tests exist, commonly used is the Chi-squared test. For this test, if the p-value $\geq 0.10$, then the data indicates that the studies are homogeneous. If the $\mathrm{p}$-value $<0.10$, the studies are understood as heterogeneous. ${ }^{4,5}$ The presence or absence of heterogeneity influences the method used to 'combine' data. If heterogeneity is absent, then the analysis employs a fixed effects model. This model assumes that the size of treatment effect is the same across all studies and the variation seen between studies is simply attributed to chance.
If heterogeneity is present, a random-effects model is used. This model assumes that the treatment effects actually vary between studies. ${ }^{9}$ Because some tests fail to detect heterogeneity, a discussion of heterogeneity and its potential effects usually accompanies summary estimates allowing readers to adequately assess results of the meta-analysis. ${ }^{4,5}$

\section{Publication Bias}

Meta-analysis also allow for the detection of publication bias. This type of bias occurs when included studies are not representative of all studies conducted to answer the question of interest. Often, studies depicting negative results or nonsignificant results are not published and may not be used in the meta-analysis. ${ }^{8}$ Meta-analysis presents two options of addressing this problem. First, most analysts attempt to include unpublished works. Second, metaanalysis can use a statistical technique to estimate the extent of publication bias in the results. ${ }^{5}$ Publication bias exists when unpublished studies yield different results compared to published studies. Unpublished studies are likely to be smaller and to have found no association between risk factor or treatment and the outcome of interest. If there is publication bias, then a strong association should exist between a study's sample size and results. ${ }^{5}$ This association can be measured using Kendall $\tau$ or by using a funnel plot. In the absence of publication bias, a plot of the standard error $v s \log$ of outcome measure (i.e. odds ratio and relative risk) should have a funnel or bell shape. When publication bias is present, the plot is asymmetrical and truncated in a corner. $^{2}$ In this manner, authors and readers are able to ascertain the validity of results presented.

\section{Sensitivity Analysis}

The findings of a meta-analysis are affected by differences in selection, inclusion and aggregation of data. In order to detect these influences, meta-analysts carry out sensitivity analyses. This analysis explores the ways in which the findings are changed by varying the approach to combining the data. For example, analysts can examine how consistent the results are across various subgroups, possibly defined by patient group, type of intervention or study setting. ${ }^{9}$

\section{Limitations}

Meta-analyses offer a systematic and quantitative approach to synthesizing evidence. However, one of the major pitfalls of this technique is that the quality of results is strongly based on the included studies. In order to ensure high quality, the search for studies must be comprehensive, appropriate checklists must be used to assess quality, publication bias must be assessed, appropriate methods must be used to 
calculate the summary effect size, and heterogeneity must be tested for. If these methodological aspects are appropriately addressed, meta-analysis can be a significant asset in answering important therapeutic questions.

\section{Conclusion: Is Meta-analysis a Sound Science?}

Meta-analyses allow for the quantitative combination of eligible studies to increase sample size, correspondingly improving the precision of summary estimates. Inherent in the proper conduct of a meta-analysis is an opportunity to check for bias and ascertain methodological rigor of the studies included and correspondingly of the meta-analysis itself. If all steps outlined are followed properly and authors remain transparent regarding the design and conduct of the meta-analysis, this technique provides an excellent and scientifically sound means of synthesizing evidence.

\section{REFERENCES}

1. Akobeng AK. Understanding the systematic reviews and metaanalysis. Arcg Dis Child 2005;90:845-48.

2. Alderson P, Green S. Publication bias. The Cochrane collaboration. Retrieved from: http://www.cochrane-net.org/ openlearning/html/mod15-3.htm 2002.

3. Cook DJ, Mulrow CD, Haynes RB. Systematic reviews: Synthesis of best evidence for clinical decisions. Ann Intern Med 1997;126:376-80.

4. Egger M, Smith GD, Phillips AN. Principles and procedures BMJ 1997;315:1533-37.
5. Grady D, Hearst N. Utilizing existing databases. In: Hulley SB, Cummings SR, Browner WS, Grady DG, Newman TB (ed). Designing Clinical Research. Philadelphia: Wolters Kluwer 2007;207-11.

6. Juni P, Altman GD, Mathias E. Assessing the quality of controlled clinical trials. BMJ 2001;323:42-46.

7. Shachar M. The preferred method of choice for the assessment of distance learning quality factors. The international review of research in open and distance learning. Meta-analysis 2008;9(3):1-15.

8. Sohani ZN, Karlsson J, Bhandari M. Special designs: Systematic reviews and meta-analyses. In Karlsson J, Marx RG, Nakamura N, Bhandari M. (Ed). A Practical Guide to Research: Design, Execution, and Publication. New York: Elsevier 2011;39-45.

9. Crombie I, Davies H. (2009). What is meta-analysis? Retrieved from http://www.adipofat.com/adipofat/docs/Meta-Analysis.pdf

10. Ioannidis JP, Lau J. Pooling research results: Benefits and limitations of meta-analysis. Jt Comm J Qual Improv 1999;25(9): 462-69.

\section{ABOUT THE AUTHOR}

\section{Zahra Sohani}

Division of Orthopedic Surgery, McMaster University, Canada

\section{CORRESPONDING AUTHOR}

Zahra Sohani, Division of Orthopedic Surgery, Hamilton General Hospital, RM CI-104, DBCVSRI Building, 237 Barton Street East Hamilton, ON, L8L 2X2, Canada, e-mail: sohaniz@mcmaster.ca 